\title{
Co-Occurrence of Seizure and Chorea in a Patient with Nonketotic Hyperglycemia
}

\author{
Sun J. Chung Jae-Hong Lee Sang-Ahm Lee Young J. No Joo-Hyuk Im Myoung C. Lee \\ Department of Neurology, Asan Medical Center, University of Ulsan College of Medicine, Seoul, Republic of Korea
}

Dear Sir,

Chorea and striatal hyperintensity on $\mathrm{T}_{1}$-weighted MRI associated with nonketotic hyperglycemia (NKH) have been recognized as a peculiar disorder [1,2]. It tends to occur in elderly people. Most reported cases are in people of east Asian origin, which suggests a possible genetic disposition to the disorder [3]. The etiological relationship between chorea and striatal hyperintensity, and the pathogenesis of striatal lesions remains unknown. Seizures associated with NKH have also been reported $[4,5]$. However, the coexistence of seizure and chorea in a patient with $\mathrm{NKH}$ has not been previously reported. We describe here a patient who concurrently developed seizure and chorea associated with NKH.

\section{Case Report}

A 78-year-old woman was admitted because of involuntary movement, intermittent confusion and visual hallucinations for 10 days. The patient had suffered from borderline diabetes mellitus for 8 years, which had been intermittently controlled with an oral hypoglycemic agent. There was no history of hypertension, trauma, infection, cerebrovascular disease or exposure to neu-

Supplementary data: We attached the video of the patient to provide additional information (published online only). roleptic drugs. She had no family history of movement disorders.

On admission, she had chorea involving the left arm and leg. The chorea was continuous and could not be suppressed voluntarily, although it disappeared during sleep. It gradually worsened over the following days, involving the left face, left arm, left leg, and the right leg to a lesser degree. The patient also showed frequent, recurrent episodes of reduced attentiveness and responsiveness, rambling speech, irritability, and visual hallucinations, which consisted of several onions painted in all the colors of the rainbow.

The level of fasting blood glucose was $473 \mathrm{mg} / \mathrm{dl}$ and glycosylated hemoglobin $\mathrm{A}_{\text {Ic }}$ was $18 \%$. No ketones were detected in urinalysis. Other routine blood tests and specialized tests including thyroid test, antistreptolysin $\mathrm{O}$, rheumatoid factor, lupus anticoagulant, antiphospholipid antibody, antinuclear antibody, anti-ds-DNA, and antineutrophil cytoplasmic antibody were all normal. Neurological examination revealed only mildly decreased deep tendon reflexes and sensation of vibration on both lower legs. Her gait was markedly impaired due to severe chorea.

A brain CT performed 10 days after symptom onset showed slightly high densities in the bilateral basal ganglia (fig. 1a). The level of hyperattenuation decreased in a follow-up brain CT performed 29 days later (fig. 1b). The $\mathrm{T}_{2}$-weighted MR images performed 12 days after symptom onset showed mild hypointensity in the left striatum (fig. 1c). Serial brain $T_{1}$-weighted MRIs, obtained 12, 25, and 93 days after the onset of chorea, revealed gradual increase in the hyperintensity of the bilateral basal ganglia (fig. 1d, e, h). The lesions were not enhanced on $\mathrm{T}_{1}$-weighted MRIs using gadolinium, performed 25 days after symptom onset (fig. 1f). Gradient echo MRIs, performed 25 and 93 days after the onset of chorea, revealed a small area of hypointensity in the left basal ganglia (fig. 1g, i). Brain single photon emission tomography, performed 21 days after the onset of chorea, revealed a normal perfusion of the bilateral basal ganglia and cortex. Video-EEG monitoring, performed 2 days after admission, clearly demonstrated an abrupt electrographic onset of 14-Hz rhythmic discharges over the right temporo-occipital area upon the occurrence of visual seizures consisting of visual hallucinations in the left half of her visual field (fig. 2). The visual seizure occurred 2 or 3 times per hour and lasted for 30-40 s. Chorea was continuously present and the amplitude of choreic movement increased when seizures occurred. The clinical and electroencephalographic seizures consistently resolved with the intravenous administration of diazepam (5 mg) during the video-EEG monitoring.

\section{KARGER}

Fax +4161306 1234 E-Mail karger@karger.ch www.karger.com (c) 2005 S. Karger AG, Basel 0014-3022/05/0544-0230\$22.00/0

Accessible online at:

www.karger.com/ene
Jae-Hong Lee, MD

Department of Neurology, Asan Medical Center

388-1, Pungnap-dong, Songpa-gu

Seoul, 138-736 (Republic of Korea)

Tel. +82 23010 3446, Fax +82 2474 4691, E-Mail jhlee@amc.seoul.kr 
Fig. 1. A brain CT (a) performed 10 days after the onset of chorea shows slightly high densities in the bilateral basal ganglia. At follow-up 19 days later, a brain CT (b) reveals decreased high densities in the bilateral basal ganglia. A brain $\mathrm{T}_{2}$-weighted MRI (c) performed 12 days after symptom onset shows subtle hypointensity in the left striatum. Brain $\mathrm{T}_{1}$-weighted MRIs, obtained 12, 25 , and 93 days (d, e, and $\mathbf{h}$, respectively) after the onset of chorea, show gradually increasing hyperintensity in the bilateral basal ganglia, which was not enhanced on a gadolinium-enhanced $\mathrm{T}_{1}$-weighted $\mathrm{MRI}$ (f). Gradient echo MRIs, performed 25 and 93 days ( $\mathbf{g}$ and $\mathbf{i}$, respectively) after the onset of chorea, show a small area of hypointensity of the left basal ganglia.

Seizures completely disappeared following the correction of hyperglycemia and EEG performed 38 days after symptom onset was normalized. However, the chorea progressively worsened over the 1-month period despite the treatment with haloperidol ( $5 \mathrm{mg} /$ day) and clonazepam ( $3 \mathrm{mg} /$ day). Haloperidol was discontinued, and a trial of clozapine up to $75 \mathrm{mg}$ /day and clonazepam moderately diminished the severity of chorea over the next 4-month period.

\section{Discussion}

To our knowledge, this is the first report of the co-occurrence of seizure and chorea in a patient with NKH, clearly demonstrated with video-EEG monitoring. Focal seizures associated with NKH were first described in 1965 [4]. In our patient, ictal manifestations were frequent visual seizures, manifesting as several onions paint-

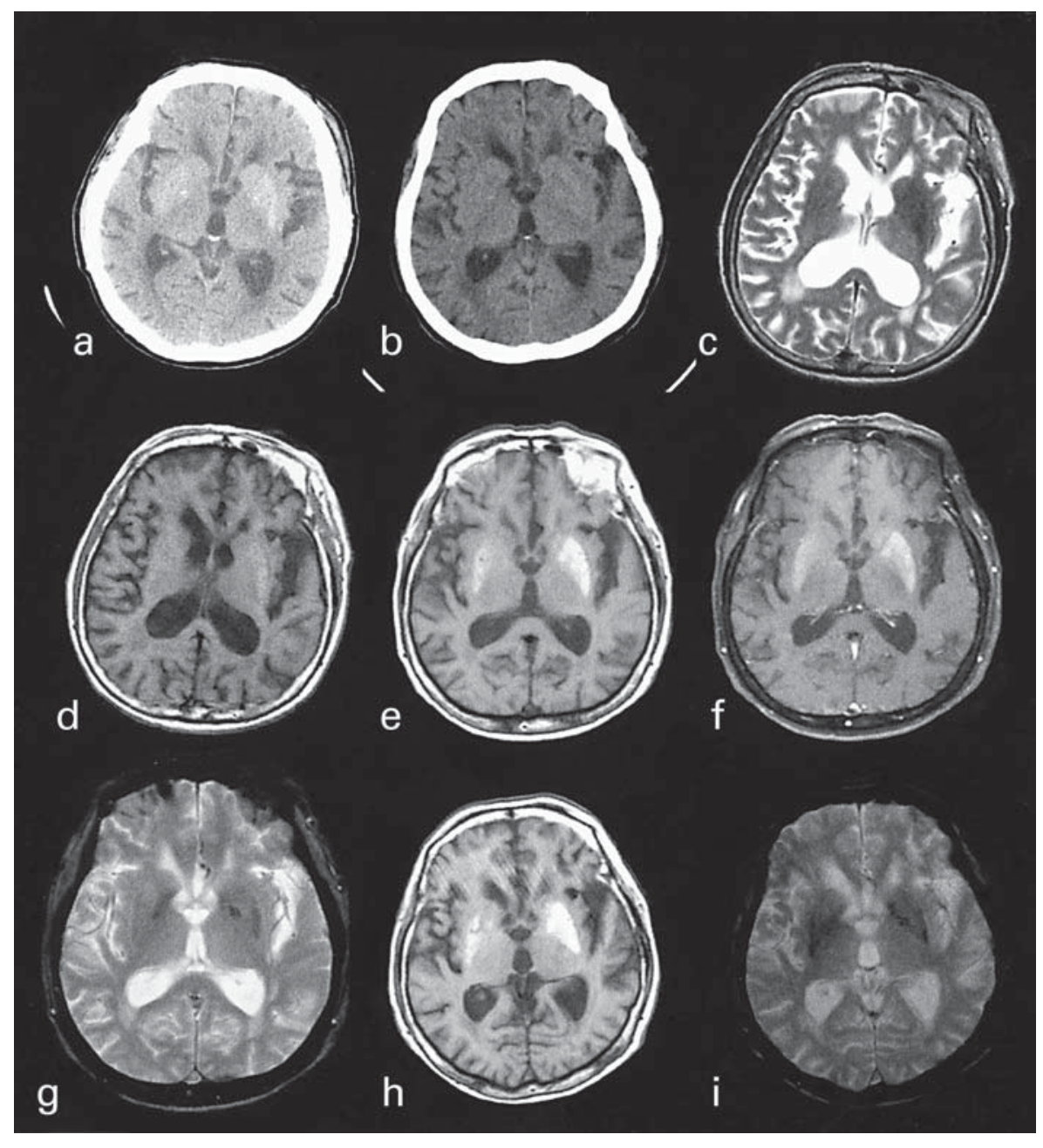

ed in all the colors of the rainbow. In accordance with previous studies [4, 5], the visual seizures of our patient completely disappeared after the correction of hyperglycemia.

Regarding the chorea of our patient, it persisted after the normalization of blood glucose abnormality, although it decreased in severity following medical treatment. Unlike seizures that have a tendency to disappear easily after the correction of hyperglycemia, chorea which persists through long-term follow-up in diabetics has been occasionally described [6]. The substantially increased striatal hyperintensity on the follow-up $\mathrm{T}_{1}$-weighted MRI may be related to the lower responsiveness to medications and the persistent chorea of our patient. Interestingly, the chorea of the left arm and leg was more violent than that of the right leg, although the hyperintensity of the left basal ganglia on the $T_{1}$-weighted MRI was more prominent than that of the right. scribes a patient with hemichorea associated with NKH caused by ipsilateral putamen hyperintensity on the $T_{1}$-weighted MRI; however, this did not offer a sufficient explanation of the pathogenic mechanism of this laterality [7].

Although the pathogenic mechanism of focal seizure or chorea in NKH remains speculative, it may be related to the lowered threshold for seizure or dysfunction of the basal ganglia due to the decreased level of $\gamma$-aminobutyric acid (GABA) [1, 2, 5, 8]. However, the fact that the chorea may persist after the correction of hyperglycemia, as in our patient and in other previously described cases [6], argues against this mechanism. Another possible mechanism is increased intracortical inhibition in the There has been only one report that de- 


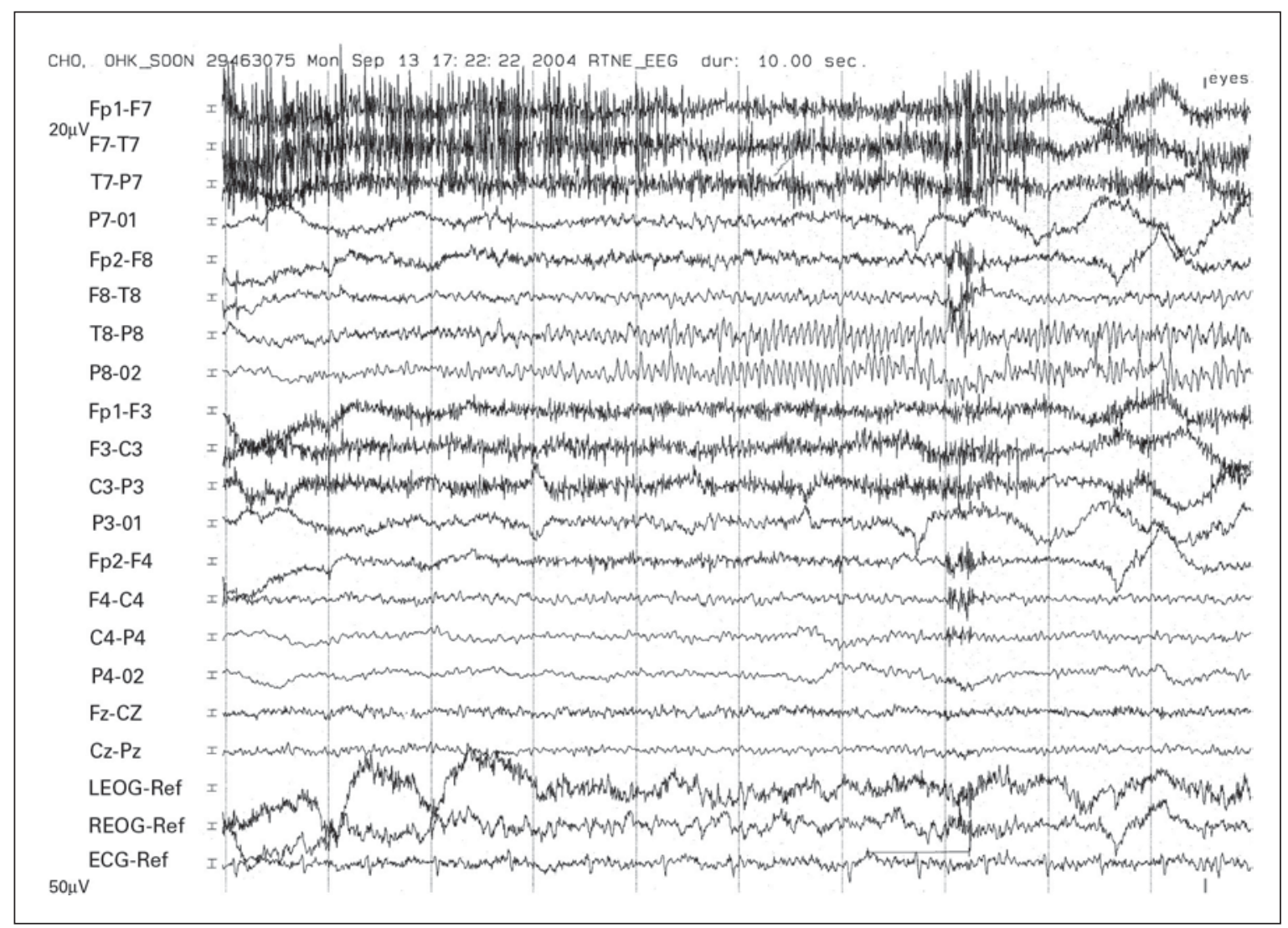

Fig. 2. Video-EEG monitoring shows electrographic onset of $14-\mathrm{Hz}$ rhythmic discharges over the right temporooccipital area (P8) upon the occurrence of visual seizure.

motor cortex contralateral to hemichorea, as suggested by Ziemann et al. [9] using transcranial magnetic stimulation.

Brain MRI findings in patients with NKH-related chorea have prompted a debate about the pathology of the lesions. In our patient, there is a striking hyperintensity in the bilateral striatum on $\mathrm{T}_{1}$-weighted MRIs. Gradient echo MRI showed a small area of hypointensity in the left putamen, which suggests that the relatively large lesions on $\mathrm{T}_{1}$-weighted MRIs were not caused by petechial hemorrhage. There has been an autopsy report on a patient with hemiballism caused by NKH [10]. Histologically, the putamen showed multiple foci of recent infarcts associated with reactive astrocyte and interneuronal response, which suggests a reaction to microinfarction. However, the cerebral tissue for histologic examination was obtained some time after symptoms had resolved and there was no occlusion of blood vessels. Therefore, the histologic correlate responsible for the findings on the brain MRI is still inconclusive. This needs to be investigated further with a pathologic study including a large number of patients.

\section{References}

1 Lee EJ, Choi JY, Lee SH, Song SY, Lee YS: Hemichorea-hemiballism in primary diabetic patients: MR correlation. J Comput Assist Tomogr 2002;26:905-911.

2 Oh SH, Lee KY, Im JH, Lee MS: Chorea associated with non-ketotic hyperglycemia and hyperintensity basal ganglia lesion on $\mathrm{T}_{1^{-}}$ weighted brain MRI study: a meta-analysis of 53 cases including four present cases. J Neurol Sci 2002;200:57-62.

3 Postuma RB, Lang AE: Hemiballism: revisiting a classic disorder. Lancet Neurol 2003;2: 661-668.

4 Maccario M, Messis CP, Vastola EF: Focal seizures as a manifestation of hyperglycemia without ketoacidosis. Neurology 1965;15: 195-206.

5 Harden CL, Rosenbaum DH, Daras M: Hyperglycemia presenting with occipital seizures. Epilepsia 1991;32:215-220.
6 Ahlskog JE, Nishino H, Evidente VG, Tulloch JW, Forbes GS, Caviness JN, Gwinn-Hardy KA: Persistent chorea triggered by hyperglycemic crisis in diabetics. Mov Disord 2001;16: 890-898.

7 Lin JJ: Ipsilateral putamen hyperintensity on $\mathrm{T}_{1}$-weighted MRI in non-ketotic hyperglycemia with hemiballism-hemichorea: a case report. Parkinsonism Relat Disord 2001;7:319321.

8 Guisado R, Arieff AI: Neurologic manifestations of diabetic comas: correlation with biochemical alterations in the brain. Metabolism 1975;24:665-679.

9 Ziemann U, Koc J, Reimers CD, Finkenstaedt M, Paulus W: Exploration of motor cortex excitability in a diabetic patient with hemiballism-hemichorea. Mov Disord 2000;15:1000_ 1005.

10 Ohara S, Nakagawa S, Tabata K, Hashimoto T: Hemiballism with hyperglycemia and striatal $\mathrm{T}_{1}$-MRI hyperintensity: an autopsy report. Mov Disord 2001;16:521-525. 